\title{
Editorial Special Topic: Enhancing Brain and Cognition via Brain Stimulation
}

\author{
Lorenza S. Colzato ${ }^{1,2,3,4} \cdot$ Roberta Sellaro $^{1}$ - Michael A. Nitsche $e^{5,6,7}$
}

Published online: 10 March 2017

(C) Springer International Publishing 2017

The idea that we can affect neural activity via electricity goes back to the late eighteenth century when Luigi Galvani showed that the muscles of dead frog legs twitched when hit by direct current on the nervous system. New techniques, such as transcranial electric stimulation (tES) and transcranial magnetic stimulation (TMS), that use electricity to modulate neuronal activity in a less invasive way than electroconvulsive therapy were recently invented. In contrast to imaging techniques, which are only correlational, by means of tES and TMS, it is possible to infer a causal relation between the stimulated brain area and a related cognitive function. This Special Issue is a forum for the exchange of novel hypotheses mainly about how tES enhances (social) cognitive processes. tES modifies brain excitability through weak, electric currents,

Lorenza S. Colzato

colzato@fsw.leidenuniv.nl

1 Cognitive Psychology Unit \& Leiden Institute for Brain and Cognition, Leiden University, Leiden, The Netherlands

2 Department of Cognitive Psychology, Institute of Cognitive Neuroscience, Faculty of Psychology, Ruhr University Bochum, Bochum, Germany

3 Institute for Sports and Sport Science, University of Kassel, Kassel, Germany

4 Institute for Psychological Research, Cognitive Psychology Unit, Leiden University, Wassenaarseweg 52, 2333

AK Leiden, The Netherlands

5 Department of Clinical Neurophysiology, Georg-August University Göttingen, Wilhelmsplatz 1, 37073 Göttingen, Germany

6 Leibniz Research Centre for Working Environment and Human Factors, Ardeystraße 67, 44139 Dortmund, Germany

7 Department of Neurology, University Medical Hospital Bergmannsheil, Bürkle de la Camp-Platz 1, 44789 Bochum, Germany and it is assumed to be a top-down technique that indirectly modulate subcortical activity through primary network changes in cortical activity.

The Special Issue comprises 8 articles with 6 mini reviews, 1 perspective and 1 opinion elaborated by 24 authors from various countries. These contributions present state-of-the art, theory-driven approaches to the study of tES to promote cognition and performance across different fields.

Schroeder and Plewnia (2017) present a nice overview of the enhancing effect of cathodal transcranial direct current stimulation (tDCS) on cognitive processes. This article suggests that the enhancing effects take place via several aspects: suppression of hyperactivity, dysfunctional network activity, noise filtering, state-dependent activity tuning (homeostatic plasticity), and distant disinhibition.

Reteig et al. (2017) review $52 \mathrm{tES}$ studies pointing to promising results in enhancing four aspects of attention that have been most extensively studied targeted to date: visual search, spatial orienting, spatial bias and sustained attention. This article discusses important suggestions for future research aiming to use tDCS as a tool to enhance or test theoretical hypotheses about attention.

Civai and Ma (2017) highlight that the enhancement of social norm compliance via brain stimulation should be carefully considered given that benchmark for deciding what is right and what is wrong is not always easy to determine in the social context.

Lavazza and Garasic (2017), within a Rawlsian theoretical framework, underline the moral reasons for some forms of regulation of non-clinical use of brain stimulation in order to "contain" eventual performance inequalities and consequent discrimination, if these new techniques were to become widespread.

Schroeder et al. (2017) review 34 tES studies showing that tDCS and transcranial random noise stimulation (tRNS) can enhance arithmetic capabilities in adult populations and could be promising tools for deviant performance populations (e.g., dyscalculia, stroke patients, children). 
Strobach and Antonenko (2017) review 31 studies showing that tDCS is a useful tool to enhance executive functions such as shifting (i.e., task switching and dual tasking), updating and inhibition.

Au et al. (2017) focus on the use of tDCS to promote longterm learning. The authors propose that the full potential of tDCS cannot be truly realized without a longitudinal design which allows for tDCS to act directly upon learning by promoting consolidation between sessions.

Lastly, Colzato et al. (2017) suggest that brain stimulation techniques seem to be useful in fine-tuning crucial aspects of competitive sports such as speeding up the learning rate of specific motor skills.

The included articles cover multiple aspects of the ongoing research in the brain stimulation field and allow an appreciation of the difficult task ahead in fully understanding the complexity of how to enhance brain and cognition via brain stimulation.

\section{References}

Au, J., Karsten, C., Buschkuehl, M., \& Jaeggi, S. M. (2017). Optimizing transcranial direct current stimulation protocols to promote long- term learning. Journal of Cognitive Enhancement. doi:10.1007/ s41465-017-0007-6.

Civai, C., Ma, I. (2017). The enhancement of social norm compliance: prospects and caveats. Journal of Cognitive Enhancement. doi:10. 1007/s41465-017-0009-4

Colzato, L. S., Nitsche, M. A., \& Kibele, A. (2017). Noninvasive brain stimulation and neural entrainment enhance athletic performance- - a review. Journal of Cognitive Enhancement. doi:10.1007/s41465016-0003-2.

Lavazza, A., \& Garasic, M. D. (2017). How non-invasive brain stimulation might invade our sphere of justice. Journal of Cognitive Enhancement. doi:10.1007/s41465-017-0008-5.

Reteig, L.C., Talsma, L.J., van Schouwenburg M.R., \& Slagter, H.A. (2017). Transcranial electrical stimulation as a tool to enhance attention. Journal of Cognitive Enhancement. doi:10.1007/s41465-0170010-y.

Schroeder, P. A., \& Plewnia, C. (2017). Beneficial effects of cathodal transcranial direct current stimulation (tDCS) on cognitive performance. Journal of Cognitive Enhancement. doi:10.1007/s41465016-0005-0.

Schroeder, P. A., Dresler, T., Bahnmueller, J., Artemenko, C., Kadosh, R. C., \& Nuerk, H. C. (2017). Cognitive enhancement of numerical and arithmetic capabilities: a mini-review of available transcranial electric stimulation studies. Journal of Cognitive Enhancement. doi:10. 1007/s41465-016-0006-z.

Strobach, T., \& Antonenko, D. (2017). tDCS-induced effects on executive functioning and their cognitive mechanisms: a review. Journal of Cognitive Enhancement. doi:10.1007/s41465-016-0004-1 